\title{
Economía de la vida. Aportes de estudios feministas y de género
}

\author{
Daniela Pessolano \\ Consejo Nacional de Investigaciones Científicas y Técnicas (CONICET), \\ Mendoza, Argentina \\ Email: dpessolano@mendoza-conicet.gob.ar
}

\begin{abstract}
Resumen $^{1}$ : Abordamos primero algunos aspectos conceptuales sobre la economía orientada a la reproducción de la vida, puntualizando luego en las contribuciones de los estudios feministas y de género al entendimiento de la economía, la reproducción y de su implicancia mutua. Construimos un recorrido que parte de la introducción de la categoría de reproducción a los estudios feministas y cuyo transcurso se define por el interés de las distintas pensadoras respecto de las prácticas de trabajo de las mujeres y de los problemas ligados a su posición social. Este enfoque, que retoma la experiencia femenina, constituye al mismo tiempo una ruptura epistemológica respecto de la mirada científica dominante del varón blanco burgués y un aporte teórico significativo que amplía la noción de trabajo, el alcance de la economía y reposiciona la reproducción social como categoría de análisis.
\end{abstract}

Palabras claves: Género, economía, reproducción, cuidado.

\section{Life economy. Contributions of feminist and gender studies}

\begin{abstract}
We adress, in the first place, some conceptual aspects of the economy oriented to life reproduction, and, in the second place, we emphasize on contributions of feminist and gender studies to understanding economy, reproduction and their mutual implications. The analysis starts with the inclusion of the "reproduction" category on the feminist studies and focuses on the interest of various authors regarding working practices of women and problems linked to their social position. This approach that considers the female experience constitutes both an epistemological rupture with the dominant scientific perspective of the white bourgeois man, and a significant theoretical contribution to extend the work concept, the scope of economy and repositions social reproduction as category analysis.
\end{abstract}

Keywords: Gender, economy, reproduction, care.

\section{Economia da vida. Aportes de estudos feministas e de gênero}

Resumo: Neste artigo abordamos em primeiro lugar alguns aspectos conceituais sobre a economia orientada à reprodução da vida, salientando logo nas contribuições dos estudos feministas e de gênero ao entendimento da economia, a reprodução e de sua implicância mutua. Construímos um percorrido que parte da introdução da categoria reprodução aos estudos feministas e cujo transcurso se 
define pelo interesse das distintas pensadoras a respeito das práticas de trabalho das mulheres e dos problemas ligados a sua posição social. Este enfoque que retoma a experiência feminina, constitui ao mesmo tempo uma ruptura epistemológica a respeito do olhar científico dominante do varão branco burguês e um aporte teórico significativo que estende a noção de trabalho, o alcance da economia e que reposiciona a reprodução social como categoria de análise.

Palavras-chaves: gênero, economia, reprodução, cuidado.

\section{Introducción}

En este artículo nos propusimos como objetivoreflexionar acerca de las relaciones entre economía y reproducción a la luz de los aportes de los estudios feministas y de género. Para ello abordamos algunos aspectos conceptuales sobre la economía empírica, es decir, orientada a la reproducción cotidiana de la vida, puntualizando luego en las contribuciones de los estudios feministas y de género al entendimiento de la economía, la reproducción y de su implicancia mutua.

Nos enfocamos en temáticas de género/feministas porque creemos que contribuyen enormemente a (re)plantear un problema sustancial, referido a la necesidad de disponer de herramientas analíticas para identificar la diversidad de actividades, actores/as y procesos económicos en las distintas sociedades, ya que sin estas herramientas la diversidad se vuelve invisible ante los ojos del/de la observador/a.

La economía no constituye una esfera autónoma y separada de la política y la cultura,desencajada de la vida. Se expresa en prácticas diversas de actores y actoras sociales que poco se parecen alhomo economicus de la teoría neoclásica y su racionalidad utilitarista.Siguiendo a Polanyi (1976),la economía se incrusta y enreda en instituciones típicamente identificadas como económicas -por ejemplo las instituciones monetarias- pero también en otras entendidas como "no económicas"-el parentesco, la religión y el gobierno-. A su vez este proceso de institucionalización del proceso económico varíasegún los tiempos y los lugares (Polanyi 1976).

Con la intención de traer de nuevo la economía a la vida de la mano de las investigaciones feministas y de género, estructuramos el presente escrito de la siguiente manera:en una primera seccióntraemos a consideraciónlas dificultades del pensamientoeconómico ortodoxoasí como de corrientes críticas -la economía marxista- para captar la "otra economía” orientada a la reproducción material de la vida,aun cuandodistintos pensadores han advertido de su existencia desde la Antigua Grecia.No obstante este contexto general,existen enfoques alternativos que ponen la reproducción de la vida -no sólo humana- en el centro. Los estudios feministas y de género constituyen un ejemplo de estas miradas alternativas ya queal recuperar las prácticas de trabajo de 
las mujerescolaboran con lacomprensión más acabada de la vida económica.

De esta manera la segunda sección se dedica a realizar una revisión dealgunos aportes teóricosde los estudios feministas y de género al entendimiento de lo económico, la reproducción y de su implicancia mutua.Para ello desarrollamos un recorrido particular cuyo eje ordenador es temporal y parte temáticamente de la recuperación de la categoría marxista "reproducción” como puntapié inicial para pensar el trabajo y la posición de las mujeres en las sociedades no sólo capitalistas. La mayoría de las pensadoras a las que aludimos se enfocan en reflexionar sobre la reproducción de la mano de obra, sin embargo y esta es su gran contribución, muestran constantemente el vínculo que existe con la reproducción del sistema económico.

Es preciso advertir, por un lado,que por las características del recorrido quedaron fuera temáticas de los estudios feministas y de géneromuy interesantes referidas a lo económico como por ejemplo el cuestionamiento a los preceptos fundantes de la economía neoclásica, los aportes del feminismo ambiental y ecofeminismo que incorporan el vínculo con la naturaleza,los esfuerzos por contabilizar la participación del trabajo no remuneradoen las cuentas nacionales y la integración de la economía feminista aproyectos de sociedad más amplios como el Buen Vivir/ Vivir Bien, en marcha en Ecuador y Bolivia² .

Por otro, advertimos que si bien usamos un criterio temporal para organizar el artículo, es no evolucionista, ya que consideramos que las discusiones del feminismo marxista y socialista cobran relevancia en el contexto de los renovados procesos de acumulación por despojo que se desenvuelven desde la instauración del capitalismo neoliberal.

En el último apartado desarrollamos ya con nuestras palabras algunas reflexiones finales respecto de lo que consideramos son los quiebres fundamentales que generan los estudios feministas y de género para pensar la economía y la reproducción.

\section{¿La reproducción de la vida también es economía?}

Sí. La reproducción de la vida también es economía sin embargo el aprovisionamiento por parte del común de la gente de medios materiales de subsistencia, no ha constituido una preocupación de la disciplina económica (Ruiz, 1999, Carrasco, 2003). En ella ha primado el pensamiento ortodoxo históricamente dominante y encarnado hoy en la vertiente neoclásica que reduce la economía a las actividades mercantiles y la riqueza a la masa de mercancías que produce una sociedad (Coraggio, 2009). El marxismo que emerge como corriente económica heterodoxa que busca objetivar la manera en que se reproduce la sociedad capitalista con el fin revolucionario de trascenderla, deposita en el trabajo productor de mercancías el motor de la acumulación secundarizando aquel otro trabajo dedicado a la producción 
de valores de uso. Ambos posicionamientos, por una u otra razón, para legitimarla o combatirla, tienen su foco en la reproducción del capital, descuidando la reproducción de la vida.

Aun cuando la definición de economía ha estado ligada a estas tradiciones de pensamiento donde prima el interés por la reproducción del capital, "la economía empírica” ha sido descubierta en tiempos inmemorables (Polanyi, 1976). Ya Aristóteles se refería a dos tipos de economía, vinculadas pero diferentes: la ciencia doméstica enfocada en la satisfacción de las necesidades básicas, donde la adquisición de riquezas tiene límites principalmente morales y la ciencia del adquirir que tiene por objeto el dinero mismo (Ruiz, 1999).

Mucho más cercano en el tiempo Karl Polanyi, en su obra La gran transformación. Crítica del liberalismo económico(1989) [1944] diferencia dos significados del término económico: uno formal y otro sustantivo. El primero deriva del “... carácter lógico de la relación medios fines, tal como aparece en palabras como ‘económico’ (barato) o ‘economizar’ (ahorrar)” (Polanyi, 1976: 155) y el segundo refiere al “...proceso instituido de interacción entre el hombre y su medio ambiente, que tiene como consecuencia un continuo abastecimiento de los medios materiales que necesitan ser satisfechos” (Ibid:159). Solo el significado substantivo, desde su perspectiva, permite desarrollar conceptos sobre los sistemas económicos empíricos. El significado formal, en cambio, puede ser aplicado únicamente a las sociedades estructuradas por un sistema de mercado (Ibid).

Edward Palmer Thompson (1979) historiador marxista muy interesado por aspectos culturales, al abordar la disciplina del tiempo que se instaura en el tránsito al capitalismo industrial en Inglaterra, identifica dos modalidades de concebir el tiempo de trabajo -que a su vez se corresponden con dos tipos de economía-. Una primera y más antigua, descripta como "orientación al quehacer” presente en sociedades campesinas, en industrias locales pequeñas y en economías domésticas aun en la actualidad, se caracteriza porque el ritmo de trabajo lo imprimen los ciclos naturales y los mismos procesos de trabajo. La orientación al quehacerrefiere tanto al pescador que debe integrar su vida con las mareas como a aquella mujer que cuida de niños pequeños y debe responder a sus necesidades vitales (Thompson 1979). La segunda modalidad es el "tiempo regulado", donde el tiempo comienza a convertirse en dinero y el dinero del patrón (Ibid). El trabajo en el contexto de los procesos de industrialización es contratado y medido en jornadas. “...el patrón debe utilizar el tiempo de su mano de obra y ver que no se malgaste: no es el quehacer el que domina sino el valor del tiempo al ser reducido a dinero. El tiempo se convierte en moneda: no pasa sino que se gasta” (Ibid: 247).

Existe entonces una economía fundamental que ha permanecido oculta,donde la gente se orienta a la satisfacción de necesidades materiales básicas como la alimentación y la vivienda y que poco tiene que ver con el homo economicus de la teoría neoclásica y su racionalidad utilitarista. A 
propósito de esta economía, hoy en Latinoamérica es posible dar cuenta de una diversidad de perspectivas y desarrollos teóricos que con pretensiones de cambio social, ponen la reproducción de la vida -no sólo humana- en el centro, cuestionando los preceptos de la economía neoclásica y marxis$\operatorname{ta}^{3}$ y destacando la relevancia de los aspectos culturales y sociales.Estos marcos interpretativos en gran medida se apoyan y recuperan prácticas concretas de distintos actores sociales: los estudios campesinos que desentrañan las particularidades de la economía campesina (Chayanov, 1974, Bartra, 1982, 2006; Archetti y Stolen, 1975), los desarrollos del Buen Vivir que revalorizan las cosmovisiones indígenas para replantear el vínculo entre los/as sujetos/as y del hombre con la naturaleza (Acosta y Martínez, 2009, Gudynas, 2011, Gudynas y Acosta, 2011), las experiencias históricas de mutualismo, cooperativismo, trueque de sectores populares urbanos que sirven para pensar que una economía social y solidaria es posible (Coraggio, 2009, 2011). En similar medida las prácticas de trabajo de las mujeres, las preguntas por su posición en la sociedad y la concentración femenina en tareas reproductivas y no remuneradas (Benería, 2005) han colaborado a una comprensión más acabada de la vida económica y de su vínculo con la reproducción social. Este tema puntual abordaremos en el próximo apartado.

\section{Estudios feministas y de género. Sus aportes al entendimiento de lo económico, la reproducción y de su implicancia mutua}

Ciertamente, las contribuciones a la comprensión de lo económico y su relación con la reproducción no provienen sólo de economistas detractoras del pensamiento económico único, sino también de epistemólogas, antropólogas y sociólogas, hecho que confirma la fecundidad de las miradas multidisciplinarias sobre un mismo objeto, en este caso los procesos económicos.

Desde la década de 1970, activistas y pensadoras del campo del feminismo y de los estudios de género han confluido en rescatar de la teoría marxista el concepto de relaciones de reproducción (Comas D’ Argemir, 1995) y con ello propiciaron una serie de debates que han devenidocon el tiempo en alternativas analíticas interesantes. Aun cuando estas reflexiones se preguntan específicamente por la posición de las mujeres en la sociedad y por sus prácticas económicas,ensimultáneo replantean el alcance de lo económico, de la noción de trabajo y reposicionan los procesos de reproducción social.

La recuperación de los escritos de Marx y Engelsllevaron a algunas pensadoras como Karen Sacks (1979) y Eleanor Leacock (1978)a abandonar las explicaciones universalistassobre los orígenes de la subordinación de la mujer (Ortner, 1979, Rosaldo, 1979) para comenzar a interpretar dicha opresión como un producto histórico puntualmente ligado a la división del tra- 
bajo social que se instauraba con el surgimiento de la propiedad privada (Comas D’ Argemir, 1995) y se caracterizaba por la diferenciación de un trabajo desarrollado en el hogar productor de valores de uso y otro, productor de valores de cambio desenvuelto en el marco de las relaciones de producción. En esta línea, incorporaron los conceptos de producción y reproducción, presuponiendo la existencia de una relación directa entre las formas de propiedad y la estructura de la producción, y la condición de la mujer.

Paralelamente se producían una serie de debates teóricos, ligados al movimiento feminista en diálogo conla tradición marxista, sobre el trabajo domésticoen el contexto de las sociedades industrializadas, los cuales eran motivados por las condiciones de invisibilización del trabajo femenino y la concentración de las mujeres en tareas reproductivas no remuneradas (Benería, 2005).

La importancia atribuida por Marx a la producción de valores de cambio y la forma en que medía el valor de la fuerza de trabajo, se encontraban en el centro de las discusiones.Por una parte y recuperando la Teoría del Valor Trabajo de Adam Smith, para Marxla plusvalía constituye el motor de la acumulación, de la reproducción de las relaciones capitalistas(Marx, 1946), de allí que le otorga al trabajo productor de mercancíasla mayor centralidad.

Por otra, equipara el valor de la fuerza de trabajo al valor de los medios de subsistencia necesarios para la conservación del individuo que posee esa mercancía. Estos medios -alimentación, mobiliario, vestido, calefacción, vivienda, educación- deben ser suficientes para mantener al trabajador en su condición normal de vida así como a su descendencia (Ibid), sin embargo, al referirse a dichos medios omite los procesos de trabajo que hacen factible que las materias primas se conviertan en elementos consumibles, es decir, omite el trabajo doméstico que implica la elaboración de los alimentos, el mantenimiento de la vestimenta o la socialización de los futuros trabajadores/as. Gardiner (2005) explica al respecto:

“...esto se debe a que el suyo era un análisis [el análisis de Marx] de un modo de producción capitalista puro, en el cual las únicas relaciones productivas eran las del trabajo asalariado realizado para el capital. Por lo tanto, consideraré que el valor de la fuerza de trabajo se refiere al valor de las mercancías compradas por el salario y consumidas por la familia del trabajador" (p. 100).

Luego de la conformación de las sociedades industriales y por la importancia atribuida a la producción de mercancías, se produce una identificación simbólica entre la concepción de trabajo y el trabajo asalariado/ empleo, acompañada a su vez de la distinción binarista entre el ámbito laboral y familiar (Todaro, 2004). Como contrapartida, el trabajo productor de valores de uso, el trabajo doméstico, queda subvalorado y su análisis omitido (Gregorio Gil, 2011) lo que carrea la invisibilización del rol económi- 
co de las mujeres a quienes se les reservala responsabilidad casi exclusiva de dicho trabajo. Por esta razón gran parte de las producciones teóricas de los años 1970- 1980 se orientaron a la ampliación del concepto restringido de trabajo y a sostener la centralidad del trabajo doméstico para la reproducción de la sociedad capitalista.

En esta línea de análisis Gardiner (2005) destaca que si bien el capitalismo trajo consigo la dependencia de los sectores trabajadores respecto de la venta de su fuerza de trabajo, con el consecuente despojo de sus medios de subsistencia, el sistema salarial nunca pudo satisfacer por completo las necesidades de estos sectores, debiendo conservar el trabajo doméstico para realizar una parte importante de la reproducción y la conservación de la fuerza de trabajo. Es decir que el trabajo doméstico termina contribuyendo a la preservación del sistema capitalista manteniendo el trabajo necesario o el valor de la fuerza de trabajo a un nivel más bajo que el nivel efectivo de subsistencia de la clase trabajadora (Gardiner, 2005, Molyneux, 2005).

Mariarosa Dalla Costa (1972) también referente de estos debates, explica que el advenimiento del capitalismodestruyó la unidad entre familia, comunidad y producción concentrando la producción social básica en la fábrica y la oficina, al mismo tiempo que separaba al hombre de la familia explotándolo a través del salario.

Si bien reconoce la claridad de Marx al objetivar la manera en que el capital domina y se desarrolla a costa del salario, identifica que no ha podido explicar la forma en que a través del salario se produce la explotación de los trabajadores no asalariados. Dalla Costa afirma:

“...esta explotación ha sido aún más efectiva porque la falta de un salario la ocultaba. Es decir, el salario controlaba una cantidad de trabajo mayor que la que aparecía en el convenio de la fábrica. En lo que respecta a las mujeres, su trabajo parece un servicio personal fuera del capital" (Ibid: 5).

En línea con lo anterior, entiende que Marx tampoco ha logrado dimensionar las particularidades de la producción de la fuerza de trabajo y explica que esta mercancía es producida fundamentalmente por el trabajo de las mujeres el cual reconoce como trabajo productivo. En sus palabras:

"Se afirma con frecuencia, dentro de la definición del trabajo asalariado, que las mujeres que hacen trabajo doméstico no son productivas. De hecho, lo cierto es precisamente lo contrario, si se piensa en la enorme cantidad de servicios sociales que la organización capitalista transforma en actividad privatizada descargándolos en las espaldas de las amas de casa” (Dalla Costa, 1972: 9).

El feminismo socialista que propició gran parte de estos derroteros ha recibido con el tiempo cuestionamientos por sus dificultades para analizar simultáneamente las valoraciones e ideologías culturales que hacen a la 
diferencias entre hombres y mujeres (Moore, 1991) y porque centraron su atención en el modo de producción capitalista (Rodríguez Enríquez, 2005).Una crítica contundente es la de Heidi Hartmann quien al examinar las relaciones entre marxismo y feminismo indica que el primero ha subordinado siempre al segundo y que las categorías marxistas son "ciegas al sexo" (1980:8). Esta autora observa que tanto marxistas como feministas marxistas han pretendido analizar la opresión de las mujeres en conexión prioritaria con la producción, por tanto, la reflexión acerca de la posición de la mujer ha quedado subsumida a la relación del obrero con el capital (Hartmann, 1980). Luego afirma que las causas de la opresión de las mujeres no se encuentran precisamente en el capital y en la propiedad privada, sino que la vía analítica correcta debe articular la relación entre patriarcado y capitalismo (Hartmann, 1980).

No obstante estas críticas,constituyen aportesdestacados que lograron problematizar la posición de las mujeres en la división del trabajo y mostrar que los análisis económicos del marxismo eran inadecuados para comprender una parte importante del trabajo que estas realizaban dedicado a la producción y reproducción de una mercancía particular y central: la fuerza de trabajo. Conjuntamente efectuaron una importante contribución teórica al evidenciar que la acumulación por parte de los sectores capitalistas sólo era posible a expensas del trabajo no remunerado desarrollado por las mujeres en los hogares.

Además, la bibliografía que se centró en reflexionar puntualmente sobre la reproducción y el lugar de la mujer en ella, favoreció el desarrollo de la teoría de las relaciones de reproducción iniciada por Marx (Comas D' Argemir, 1995, Narotzky, 2004). En este sentido, pensadoras como Edholm, Harris y Young (1982) denuncian que el concepto de reproducción ha sido abordado de manera problemática por algunos marxistas destacados como Meillassoux y Althusser. Sostienen asimismo que se produjo una lectura en exceso simplista que integra muchos elementos analíticamente distintos, lo que genera una confusión en cuanto al contenido de la reproducción (Edholm, Harris y Young, 1982).Por tal motivo, sin negar los aportes del marxismo, desglosan la categoría reproducción con la finalidad de hacerla operativa y de otorgarle potencialidad explicativa (Harris y Young,1981). En vez de separar un nivel económico y otro reproductivo, diferencian tres niveles de abstracción teórica de la reproducción: a nivel más amplio la reproducción social de las condiciones que sostienen una formación social particular,luego la reproducción de la mano de obraque refiere al mantenimiento cotidiano de trabajadores presentes y futuros y a la asignación de agentes a posiciones en el proceso productivo, y por último, la reproducción biológica de las poblaciones humanas (Ibid). Cada nivel de la reproducción tiene implicancias particulares para las mujeres y se encuentra mutuamente comprometido con los otros niveles (Ibid).

Por su parte, Benería (1981) aborda la relación entre procesos productivos y reproductivos, argumentando que el control de las actividades reproductivas es una condición indispensable para la perpetuación del sis- 
tema social, y con él, para la reproducción de las desigualdades en el acceso a los recursos productivos en las sociedades de clases. Con este supuesto la autora le otorga un lugar de importancia a la dimensión reproductiva de la economía que hasta el momento sólo se atribuía a la dimensiónproductiva, particularmente a las relaciones de producción.

La reproducción del sistema social de clases, para Benería (1981), requiere de la identificación precisa de los sujetos que serán destinatarios del acceso y el control de los recursos económicos, más considerando que los recursos transmitidos son cualitativa y cuantitativamente distintos de acuerdo a cada grupo. Esto, que debe asegurarsede una generación a otra, se sirve de un conjunto de mecanismos derivados de los procesos reproductivos y con ellos del control de las actividades de las mujeres (Ibid). Las consecuencias entonces para las mujeres son en primer lugar, el control de sus actividades reproductoras y de su sexualidad, en gran medida mediante la institución del matrimonio pero también a través de la reclusión, la mutilación genital y las restricciones a su movilidad (Ibid). En segundo lugar, y en íntima relación con el rol de las mujeres en la reproducción biológica, el cuidado de niños y niñas y las tareas domésticas se convierten en un dominio exclusivamente femenino (Ibid).

Por tanto, el control que sujeta a las mujeres a la reproducción biológica y de la fuerza de trabajo lleva a Benería (1981) a sostener la imposibilidad de entender la participación femenina en la producción si no es teniendo en cuenta su papel reproductivo.

Una autora que no podemos dejar de nombrar, que destaca al igual que Benería (1981) la importancia de los procesos reproductivos y del control de las mujeres para la conformación y sostenimiento del sistema capitalista, es Silvia Federici con su obra Calibán y la Bruja. Mujeres, cuerpo y acumulación originaria (2011). En la misma, profundiza una línea de investigación que comenzó con Leopoldina Fortunati en los años 1970, acerca de las mujeres en la transición del feudalismo al capitalismo enfocando su atención en“la caza de brujas” que tuvo lugar en los siglos XVI y XVII en Europa y en el "Nuevo Mundo” (Federici, 2011). Su análisis se diferencia del marxista porque en vez de abordar esta transición desde el punto de vista de los asalariados de sexo masculino y poniendo el foco en la producción de mercancías, lo hizo desde el punto de vista de los cambios que introduce en la posición social de las mujeres y en la producción de la fuerza de trabajo (Ibid). Allí Federici enriquece la interpretación histórica al exponer que la acumulación primitiva además de conformar trabajadores asalariados “libres” separados de sus medios de producción, necesitó fundar una nueva división sexual del trabajo que excluye a las mujeres del trabajo asalariado productor de mercancías, las subordina a los hombres y las convierte en “...una máquina de producción de nuevos trabajadores” (Ibid: 23).Federici (2011) argumenta:

“(...) en la sociedad capitalista, el cuerpo es para las mujeres lo que la fábrica es para los trabajadores asalariados varones: el principal 
terreno de su explotación y resistencia, en la misma medida en que el cuerpo femenino ha sido apropiado por el Estado y los hombres, forzado a funcionar como un medio para la reproducción y la acumulación de trabajo” (pp.29-30).

Hasta aquí podemos identificar que las preguntas por las prácticas y experiencias de las mujeres derivaron en una forma particular de pensar la economía,la producción, la reproducción y la relación entre estos términos. Un cambio de mirada, de “punto de vista”. Esto significó en cierta medida una ruptura con un posicionamiento epistemológico histórico, aun dominante, en el que los intereses y experiencias de hombres -mayoritariamente blancos y burgueses- habían primado, favoreciendo su posibilidad de definir los problemas e hipótesis que debían ser objeto de explicación científica (Harding 1995). De allí, las pensadoras mostraron que la definición de trabajo y economía ceñida al mercado, a la producción y al salario era insuficiente, ya que las prácticas económicas de las mujeres desbordaban ampliamente los límites de dicha definición. Permitieron ver pues, que los desarrollos teóricos marxistas aportaban elementos para comprender la situación económica de las mujeres en parte, por ello comenzaron a recuperan la categoría género para vincular las construcciones en torno a lo masculino y lo femenino con las relaciones de producción capitalistas.

Con los años venideros, principalmente a partir de la primera década del s. XXI, se recupera el abordaje teórico del trabajo de las mujeres de la mano de la economía feminista ${ }^{4}$ (Rodríguez Enríquez, 2015), haciendo de este un verdadero campo de estudio. Sin embargo esta tarea se llevó a cabo a partir de un alejamiento de la categoría marxista de trabajo elaborada según referencias de mercado, lo quecondicionó durante los debates sobre el trabajo doméstico, la ampliación de este concepto -trabajo(Carrasco, 2009) ${ }^{5}$.

La bibliografía de los años '70 y '80 consideró del trabajo doméstico familiar principalmente su dimensión objetiva, es decir, su asociación con la provisión de bienes y servicios como alimentos, vestimenta, estudio, techo, entre otras. Los estudios recientes en cambio, han avanzado en clarificar que dicho trabajo presenta otra dimensión de carácter subjetiva que refiere a la satisfacción de necesidades de cuidados, relacionales y emocionales y se caracteriza porque difícilmente encuentra sustitutos en el mercado o en la provisión estatal, y porque, al igual que la dimensión objetiva es transcendental para la viabilidad del funcionamiento de la esfera mercantil capitalista (Carrasco, 2003).

Según Picchio (1994) y Carrasco (2003) lo que intenta ocultarse desde las concepciones dominantes no es el trabajo doméstico en si mismo, sino la relación que este último establece con la reproducción del sistema social, hecho que posibilita externalizar los costos originados por las actividades mercantiles y cargarlos sobre la familia, principalmente sobre sus integrantes mujeres. 
Como resultado de la (re)valorización de la dimensión subjetiva, se construyen los conceptos trabajo de cuidados y economía de cuidados (Carrasco, Borderías y Torns 2011), afirmándoseestas nuevas denominaciones en la idea de que las actividades domésticas poseen rasgos diferenciales respecto de las mercantiles.

De manera más específica entonces, el trabajo doméstico se caracteriza por su orientación al cuidado de la vida y el bienestar de las personas en el hogar e involucra indefectiblemente una alta carga afectiva a diferencia del trabajo mercantil que, en última instancia, busca la acumulación de beneficios (Picchio, 1994, Carrasco, 2009) y se desarrolla, en general, en el seno de relaciones despersonalizadas. Asimismo -el trabajo domésticoconstituye un trabajo universal que además del bienestar de las personas, en las sociedades capitalistas apunta al objetivo particular de facilitar la disponibilidad laboral de los hombres adultos del hogar para que participen del mundo público sin que las necesidades de cuidado del hogar constituyan una restricción a su actividad. Por otro lado ha sido asignado tradicionalmente a la población femenina bajo una fuerte impronta simbólica de género. Y se define por las relaciones en las que se inserta, no por las características de la tarea concreta ${ }^{6}$

(Carrasco et al., 2011). Es preciso destacar que este trabajo no se realiza sólo por afecto, al contrario, se desarrolla bajo presiones sociales, culturales, psicológicas o incluso de manera indeseada y bajo violencia física (Rodríguez Enríquez, 2005).De la misma manera, presenta desigualdades condicionadas por diferencias de etnia y clase social (Carrasco et al., 2011).

Sin embargo, Rodríguez Enríquez (2005, 2015) señala que hablar de economía de cuidados es más adecuado ya que dicho concepto enfatiza lascontribucionesdel cuidado a la producción de valor económico y además permite trazar relaciones estrechas entre la forma en que la sociedad organiza el cuidado de sus miembros y el funcionamiento del sistema económico.

La economía de cuidados se presenta como el núcleo de la reproducción social, identificadacon los procesos de reproducción de la fuerza de trabajo y se asegura mediante formasde organización social del cuidado específicas, que cada sociedad estructura. En ellas familias, Estado, mercado y organizaciones comunitarias producen y distribuyen cuidados de manera interrelacionada (Rodríguez Enríquez, 2015). Esta misma autora al analizarla organización social del cuidado en América Latina informa que existen serias desigualdades en la distribución de las responsabilidades de cuidados entre todos estos actores, viéndose recargados principalmente los hogares y, dentro de ellos, las mujeres (Ibid).

En síntesis, las autoras citadas estarían volviendo a afirmar en el fondo de la cuestión, la dificultad analítica de separar un nivel económico y otro reproductivo autónomo al proceso de producción, y la necesidad de 
revisar algunas herramientas conceptuales, fundamentalmente las que provienen de un pensamiento dualista que separa tajantemente público de privado, familia de trabajo, trabajo de ocio, producción de reproducción (Comas D’Argemir, 1995,Bosch, Carrasco y Grau, 2005).

Las contribuciones de la economía feministatrascienden el ámbito doméstico y los procesos de reproducción cotidiana de los que nos venimos ocupando en este escrito. Una de sus significativas propuestas, de carácter tanto político comoanalítico, ha sido proyectar una economía orientada ala reproducción o sostenibilidad de la vida y no a la reproducción del capital (Benería, 2005, León, 2011, Carrasco, 2009, Pérez Orozco, 2014). Este cambio de paradigma de alcance macroeconómico ypropuesta revolucionaria,suponeel tránsitode la lógica de la cultura del beneficio a la lógica de la cultura del cuidado y para elloargumenta que los tiempos que deben primar no son precisamente los de la producción capitalista (Carrasco, 2003).

"Si optamos por la vida humana (...) entonces habría queorganizar la sociedad siguiendo el modelo femenino de trabajo de cuidados: una forma discontinua de participar en el trabajo familiar que dependerá del ciclo vital de cada persona, mujer u hombre. Los horarios y jornadas laborales tendrían que irse adaptando a las jornadas domésticas necesarias y no al revés como se hace actualmente. Los tiempos mercantiles tendrían que flexibilizarse pero para adaptarse a las necesidades humanas. El resultado sería una creciente valoración del tiempo no mercantilizado, lo cual colaboraría a que el sector masculino de la población disminuyera sus horas dedicadas al mercado y fuera asumiendo su parte de responsabilidad en las tareas de cuidados directos” (Carrasco, 2003:20).

Asimismo, la cercanía entre ecologismo y feminismo económico, y específicamente en América Latina entre feminismo económico y cosmovisiones indígenas en particular andinas, han llevado a pensar la sostenibilidad de la vida en unos términos más amplios e integrales que consideran el cuidado no sólo de los seres humanos, sino de todas las formas de vida (León, 2011, 2008, Herrero López, 2013,Carrasco et al., 2011, Boschet al., 2005). Por tal motivo algunas autoras han acuñado el concepto de sostenibilidad económica, social y ecológica (Carrasco et al., 2011) y en un sentido similar Pérez Orozco define la sostenibilidad de la vida como el “...sostenimiento de las condiciones de posibilidad de vidas que merecen la pena ser vividas” (Pérez Orozco, 2014:73).

Detrás de esta idea general se sitúan dos supuestos básicos. Por un lado,la afirmación de que la vida no sería posible sin los bienes que provee la naturaleza y los trabajos de cuidados que en gran medida desarrollan las mujeres en sus hogares. Y por otro pero en íntima vinculación,la economía oficial se ha encargado sistemáticamente de invisibilizar aquellos procesoseconómicos no monetarios y no mercantiles lo que ha dado como resultado un tratamiento análogo respecto de los trabajos no remunerados 
de las mujeres y de los bienes de la naturaleza. Tanto unos como otros han sido usados por el capital sin contabilizar sus costos, bajo la definición de externalidadeconómica de oferta ilimitada (Herrero López 2013, Carrasco et al., 2011, Bosch et al., 2005).

Si bienlos enfoques analíticos feministas y de los estudios de género son variados y desde distintos posicionamientos realizan contribuciones diversas, en este texto nos dedicamos de manera un tanto escueta, a traer algunos elementos conceptuales -más que de contexto- para comprender la economíarealmente existentey su relación con la reproducción social.

Podríamos retomar la idea de Harding respecto de que los estudios de la mujer, feminista y de género fueron adquiriendo cierta madurez en un proceso que se inició “agregando mujeres y mezclándolas” en las teorías convencionales, sin alterar sus presupuestos centrales, para avanzar luego hacia desarrollos teóricos críticos que han retomado las experiencias de las mujeres en el estudio de la vida social (Harding, 1998) y con ello arribaron a análisis fructíferos de la economía en su conjunto.

\section{Reflexiones finales}

En este artículoabordamos algunos aspectos conceptuales sobre la economía orientada a la reproducción de la vida, puntualizando en las contribuciones de los estudios feministas y de género al entendimiento de la economía, la reproducción y de su implicancia mutua.Construimos un recorrido-entre los tantos posibles-que parte de la introducción de la categoría reproducción a los estudios feministas y cuyo transcurso se define por el interés de las distintas pensadoras respecto de las prácticas de trabajo de las mujeres y de los problemas ligados a su posición social. Este enfoque que retoma la experiencia femenina, constituye al mismo tiempo una ruptura epistemológica -respecto de la mirada científica dominante del varón blanco burgués- y un aporte teórico significativo que amplía la noción de trabajo, el alcance de la economía y reposiciona la reproducción social como categoría de análisis.A continuación realizamos algunos señalamientos respecto de estos aportes.

Las investigaciones feministas y de género,en primer lugar, pusieron en evidencia que los enfoques económicos neoclásicos y marxistas presentan serias dificultades para captar las contribuciones de las mujeres a la economía debido, en gran medida, a que su foco esta puesto en la producción mercantil. Estos análisis, están orientados fundamentalmente a la reproducción del capital y tienden a descuidar la reproducción de la vida humana, al tiempo que ignoran las relaciones de poder que se estructuran alrededor de las diferencias sexuales.

En segundo lugar argumentaron que la producción de mercado y con ella la acumulación capitalista es posible a expensas del trabajo doméstico no remunerado desarrollado en los hogares principalmente por parte de 
sus integrantes mujeres. En otros términos Narotzky expresa“...el análisis de cómo se producen los productos es indisociable del análisis de cómo se producen la personas...” (1995:137). Los estudios recientes redoblaron la apuesta del feminismo marxista y socialista al sostener que no sólo los bienes y servicios que brinda el trabajo doméstico son indispensables para la reproducción social general, sino también los aspectos subjetivos de este trabajo orientados ala satisfacción de necesidades de cuidado, emocionales y relacionales que producen trabajadores “aptos”para desenvolverse según las exigenciasdel mercado laboral.

De lasafirmaciones del párrafo anterior, se desprende una tercera cuestión; la dificultad de separar de manera taxativa un ámbito de producción y otro de reproducción, la estructura económica de la superestructura, la casa del trabajo,para estudiar la economía y lo imperioso de disponer de marcos analíticos superadores de esas dicotomía como los que propone la economía de cuidados y los enfoques de la reproducción social. Por tanto, desde estas perspectivas, lo económico rebasaría los límites de la producción y el mercado e involucraría las relaciones de parentesco y la satisfacción de necesidades materiales y afectivas.

Estos estudios han demostrado que la mirada sobre lo económico se enriquece y complejiza al recuperar lasprácticas de las mujeres y la categoría género, en efecto, es preciso vincular las construcciones materiales y simbólicas en torno a lo masculino y lo femenino con las relaciones de producción que se estructuran en el capitalismo.

Los enfoques económicos más difundidos,sus conceptos y categorías, no son omniexplicativos ni habilitan siempre la comprensión, por el contrario, y como sucedeademás con las prácticas campesinas e indígenas por ejemplo, en repetidas oportunidades ocultan aspectos -relaciones, procesos, actores/as- de las distintas realidades, sobre todo cuandoponen en cuestiónlos fundamentos del sistema capitalista patriarcal colonial. 


\section{Notas}

${ }^{1}$ Este artículo forma parte de una investigación en curso, desarrollada en el contexto de una tesis doctoral, titulada "Mujeres, participación económica y persistencia campesina. Un estudio de caso en tierras áridas no irrigadas del Este de Mendoza”.

${ }^{2}$ Hemos avanzado en la línea de esta última temática en el artículo "Diálogosentre feminismos y BuenVivir/VivirBien: distintas perspectivas,múltiplespuntosdeencuentro" (2013)de la Revista Confluencia Sociología,Universidad Nacional de Cuyo.

${ }^{3}$ Es preciso realizar una salvedad respecto de la economía marxista. Sus análisis estructurales permiten ver, como plantea Godelier (1976) “la lógica profunda” del funcionamiento económico capitalista. Por tanto las propuestas contemporáneas inclusive los feminismos- suelen recuperar la economía marxista con ciertas precauciones, marcando sus limitaciones analíticas e intentando superarlas. Es el caso de propuestas de autores/as como Bartra, Coraggio y Federici.

${ }^{4}$ La economía feminista surge en 1992 ligada a la Asociación Internacional de Economía Feminista, a la publicación inglesa Feminism economics (Benería 2005, Espino 2011) y al libro fundador Beyond Economic Man de Ferber y Nelson (comps.) (1993). Ha realizado aportaciones diversas entre las que se encuentra, de manera predominante, la construcción de nuevos significados para la economía y el cuestionamiento de la economía ortodoxa, principalmente de su vertiente neoclásica. En esta línea han problematizado supuestos neoclásicos fundamentales como el de racionalidad económica, el comportamiento maximizador como norma de conducta, la retórica de la eficiencia, el homo economicus como prototipo de sujeto autosuficiente, el peso de la elección en la economía (Benería 2005: 53). Asimismo en la actualidad se constituye un campo de conocimiento que encuentra en crecimiento en América Latina (Esquivel, 2010).

${ }^{5}$ Siguiendo a esta última autora, se argumentaba en los años '70 - '80 que las actividades domésticas cumplían con los requisitos del trabajo asalariado y por ello constituían trabajo: “...requerían de tiempo y energía para poder realizarse (de aquí que se le podía asignar un coste de oportunidad); formaban parte de la división del trabajo (las aportaciones de mujeres y hombres al hogar eran diferentes) y producían bienes y servicios (comida, limpieza) separables de la persona que los realizaba, es decir podían ser producidos en el mercado aunque bajo otras relaciones de producción”(Carrasco, 2009: 48).

${ }^{6}$ Las autoras proponen como ejemplo el hecho de que mientras un plato de comida preparado por un ama de casa en su hogar constituye trabajo doméstico, el mismo plato de comida elaborado en un restaurante por esa misma mujer será definido como trabajo mercantil (Carrasco et. al., 2011: 73). 
Polis, Revista Latinoamericana, Volumen 15, $N^{\circ}$ 45, 2016

\section{Bibliografía}

Acosta,A.yMartínez,E.(2009).ElBuenVivir.Unavíaparaeldesarrollo. Ed.Abya -Yala, Quito.

Archetti, E. y Stolen, K. (1975). Explotación familiar y acumulación de capital en el campo argentino. Siglo XXI, Buenos Aires.

Bartra, A. (1982). La explotación del trabajo campesino por el capital. Macehual, México.

Ídem (2006). El capital en su laberinto. De la renta de la tierra a la renta de la vida. UACM, México.

Benería, L. (1981) [1979]. Reproducción, producción y división sexual del trabajo. Mientras Tanto, $\mathrm{n}^{\circ} 6, \mathrm{p} .47-84$.

Ídem (2005). Los estudios sobre la mujer y el género en la economía: una visión histórica. En Benería. Género, Desarrollo y Globalización. Por una ciencia económica para todas las personas. Hacer editorial, Barcelona.

Bosch A., Carrasco C. y Grau E. (2005). "Verde que te quiero violeta. Encuentros y desencuentros entre feminismo y ecologismo. En Tello (comp.). La historia cuenta, El Viejo Topo, Barcelona, p. 321-346.

Carrasco C., Borderías C. y Torns T. (2011) “Introducción. El trabajo de cuidados: antecedentes históricos y debates actuales”. En Carrasco C., Borderías C. y Torns T. (eds.). El trabajo de cuidados. Historia, teoría y políticas, Catarata, Madrid, p.13-95.

Carrasco, C. (2003) [2001]. "La sostenibilidad de la vida humana: ¿un asunto de mujeres?”. En León (comp.) Mujeres y trabajo: cambios impostergables, ALAI, Porto Alegre, p. 11-49.

Ídem (2009) “Tiempos y trabajos desde la experiencia femenina”. Revista PAPELES de relaciones ecosociales y cambio global, $\mathrm{n}^{\circ} 108$, p.45-54.

Chayanov, A. V. (1974). La organización de la unidad económica campesina, Nueva Visión, Buenos Aires.

Comas D’ Argemir, D. (1995). Trabajo, Género y Cultura, la construcción de desigualdades entre hombres y mujeres. ICARIA, España.

Coraggio, J.L. (2009). “Territorio y economías alternativas”. Ponencia presentada en el ISeminario Internacional Planificación Regional Para El Desarrollo Nacional. Visiones, desafíos y propuestas, La Paz, Bolivia.

Ídem (2011). Economía social y solidaria. El trabajo antes que el capital. Ediciones Abya-Yala, Quito. 
Dalla Costa, M. (1972). “Las mujeres y la subversión de la comunidad”. En Dalla Costa, y James. El poder de la mujer y la subversión de la comunidad, Siglo XXI, México.

D’AmicoP. y Pessolano,D.(2013). “Diálogosentrefeminismosybuenvivir/ vivirbien:distintasperspectivas, múltiplespuntosdeencuentro”. RevistaConfluenciaSociología.UNCuyo, Mendoza, p. 13-35.

Edholm F., Harris O. y Young K. (1982) [1977]. “La conceptualización de la mujer”. En INEGI Estudios sobre la mujer, Coordinación General de los Servicios Nacionales de Estadística Geográfica e Informática, México, p. 344-373.

Espino, A. (2011). “Economía feminista: enfoques y propuestas”. En Sanchis (Comp.). Aportes al debate del desarrollo en América Latina. Una perspectiva feminista. Red de Género y Comercio, Buenos. Aires.

Esquivel, V. (2012). “Introducción: Hacer economía feminista desde América Latina”. En ONU Mujeres, La economía feminista desde América Latina. Una hoja de ruta sobre los debates actuales en la región, ONU Mujeres, Santo Domingo, p.24-41.

Federici, S. (2011). Calibán y la bruja. Mujeres, cuerpo y acumulación originaria. Tinta Limón, Buenos Aires.

Gardiner, J. (2005) [1975]. “El trabajo doméstico de las mujeres”. En Rodríguez y Cooper. El debate sobre el trabajo doméstico. Antología. UNAM, México.

Godelier, M. (1976) [1974]: “Antropología y economía. ¿Es posible la antropología económica?”. En Godelier (comp.) Antropología y economía. Anagrama, Barcelona, p. 279-333.

Gregorio Gil, C. (2011). “Análisis de las migraciones transnacionales en el contexto español, revisitando la categoría de género desde una perspectiva etnográfica y feminista”. Nueva Antropología, vol. XXIV, n74, p.39-71.

Gudynas,E.(2011). “Tensiones, contradiccionesyoportunidadesde ladimensiónambientaldelBuenVivir”.EnFarah I. y Vasapollo, L.(Coord.)Vivirbien:¿Paradigmanocapitalista?, CIDES - UMSAyPlural, LaPaz, p.231-246.

Gudynas, E. y Acosta, A. (2011). "La renovación de la crítica al desarrollo y el Buen Vivir como alternativa”. En Utopía y praxis latinoamericana, Maracaibo, 16 (53), p.71-83.

Harding, S. (1995). “Del problema de la mujer en la ciencia al problema de la ciencia en el feminismo”. En Harding (1995) Feminismo y ciencia, Morata, Barcelona, p.15-27. 
Ídem (1998) “¿Existe un método feminista?”. En Bartra, Eli (comp.) Debates en torno a una metodología feminista. UAM-X CSH, México DF, p. 9-34.

Harris O. Y Young K. (1981). "Engendered structures: some problems in the analysis of reproduction”. En Kahn y Llobera (eds.) The Anthropology of pre-capitalist societies. Macmillan, Londres.

Hartmann, H. (1980). “Un matrimonio mal avenido: hacia una unión más progresiva entre marxismo y feminismo”. Zona Abierta, n² 24, p.85-113.

Herrero López Y. (2013). “Feminismo y ecología reconstruir en verde y violeta”. En Sánchez Maldonado, López Castellano, Manzanera Ruiz, Miguel Juan (coord.). Medioambiente y desarrollo: miradas feministas desde ambos hemisferios, Universidad de Granada, Granada, p. 67-86.

Leacock, E. (1978). “Women's status in egalitarian society: implications for social evolution”. Current Anthropology, vol. 19, n², p.247-275.

León, M. (2006). “Algunos Desafíos para la Economía Feminista en América Latina”. En CEFEMINA. Nosotras hacemos la (otra) economía. Aportes a los debates feministas sobre la economía, CEFEMINA, San José, p.13-22.

Ídem (2009). “Cambiar la economía para cambiar la vida. Desafíos de una economía para la vida”. En Acosta y Martínez (comp.), El buen vivir. Ed. Abya-Yala, Quito.

Ídem (2011). "Redefiniciones económicas hacia el Buen Vivir: un acercamiento feminista”. En AWID. Buen Vivir: experiencias en Bolivia y Ecuador desde una perspectiva de derechos de las mujeres. Consultado en: fedaeps.org/IMG/pdf/Magdalena_Leon_buenvivir_economia.pdf

Marx, C (1946). El Capital. Tomo I. Fondo de Cultura Económica, México DF.

Molyneux M. (2005) [1979]. “Más allá del debate sobre el trabajo doméstico”. En Rodríguez y Cooper. El debate sobre el trabajo doméstico. Antología. UNAM, México.

Moore, H. (1991) “Género y estatus. La situación de la mujer”. En Moore. Antropología y feminismo, Cátedra, Madrid.

Narotsky, S. (2004). Antropología Económica. Nuevas Tendencias. Melusina, España.

Ídem (1995) Mujer, mujeres, género: una aproximación crítica al estudio de las mujeres en las ciencias sociales. CSIC, España.

Ortner, S. (1979) “¿Es la mujer al hombre lo que la Naturaleza es a la Cultura?”. En Harris y Young (comp.). Antropología y Feminismo, Anagrama, Barcelona, p.109-131. 
Pérez Orozco, A. (2014). Subversión feminista de la economía. Aportes para un debate sobre el conflicto capital-vida. Traficantes de sueños, Madrid.

Picchio, A. (1994). "El trabajo de reproducción, tema central en el análisis del mercado de trabajo”. En Borderías, Carrasco, Alemany (comp.). Las mujeres y el trabajo. Rupturas conceptuales, Fuhem-Icaria, Madrid.

Polanyi, K. (1976) [1958]. “El sistema económico como proceso institucionalizado”. En M. Godelier (comp.). Antropología y economía, Anagrama, Barcelona, p. 155 -178.

Ídem (1989) [1944]. La gran transformación. Critica del liberalismo económico. Ediciones de La Piqueta, Madrid.

Rodríguez Enríquez, C. (2005). “Economía del cuidado y política económica: una aproximación a sus interrelaciones”. Trigésima octava reunión de la Mesa Directiva de la Conferencia Regional sobre la Mujer de América Latina y el Caribe CEPAL. Mar del Plata.

Ídem (2015). “Economía feminista y economía del cuidado. Aportes conceptuales para el estudio de la desigualdad”. Revista Nueva Sociedad $\mathrm{n}^{\circ}$ 256, p.30-44.

Rosaldo, M Z. (1979). “Mujer, cultura y sociedad: una visión teórica”. En Harris y Young. Antropología y Feminismo, Anagrama, Barcelona, p.153180.

Ruiz, B. (1999). “La reproducción también es economía”. Áreas. Revista Internacional de Ciencias Sociales n²19, p.101-115.

Sacks, K. (1979). "Engels revisitado: las mujeres, la organización de la producción y la propiedad privada”. En Harris y Young. Antropología y Feminismo, Anagrama, Barcelona.

Todaro, R. (2004) “Ampliar la mirada: trabajo y reproducción social”. En Todaro y Yáñez (editoras). El trabajo se transforma. Relaciones de producción y relaciones de género. Centro de Estudios de la Mujer, Santiago de Chile.

Thompson, E.P. (1979). “Tiempo, disciplina de trabajo y capitalismo industrial”. En Thompson, E.P. Tradición, revuelta y consciencia de clase, Editorial Crítica, Barcelona, p. 239-293.

Recibido: 27.10.16

Aceptado: 01.12.16 